\title{
Determinants of parental responsibility to promote healthy eating behaviors in children: a scoping review protocol
}

\author{
Determinantes da responsabilidade parental para promover comportamentos alimentares \\ saudáveis em crianças: um protocolo de revisão de escopo \\ Factores determinantes de la responsabilidad parental para promover conductas alimentarias
}

saludables en los niños: a scoping review protocol

Received: 10/20/2021 | Reviewed: 10/28/2021 | Accept: 11/26/2021| Published: 12/02/2021

\author{
Azucena Lizalde Hernández \\ ORCID: https://orcid.org/0000-0002-7709-7141 \\ Universidad de Guanajuato, Mexico \\ E-mail: a.lizaldehernandez@ugto.mx \\ Juliana Graciela Vestena Zillmer \\ ORCID: https://orcid.org/0000-0002-6639-8918 \\ Universidade Federal de Pelotas, Brazil \\ E-mail: juzillmer@gmail.com \\ María Mercedes Moreno González \\ ORCID: https://orcid.org/0000-0002-0299-2299 \\ Universidad de Guanajuato, Mexico \\ E-mail: ma.moreno@ugto.mx \\ Josefina Valenzuela Gandarilla \\ ORCID: https://orcid.org/0000-0002-3886-4977 \\ Universidad Michoacana de San Nicolás de Hidalgo, Mexico \\ E-mail: josefina.valenzuela@umich.mx
}

\begin{abstract}
Introduction: The parental responsibility of parents regarding the feeding of children implies having a responsible job when selecting, preparing and offering food to their children; in addition, qualities of exemplary behavior such as healthy eating behavior that influences the habits that their children acquire; however, there are multiple determining factors that negatively affect it. Objective: To identify the determining factors in the parental responsibility of caregivers to promote an eating behavior in children from 6 months to 12 years old. Methods: The review will be carried out according to the methodology proposed by Hilary Arksey and Lisa O`Malley updated by The Joanna Briggs Institute (JBI) for scoping review. A comprehensive search strategy will be applied with the support of the research librarian to refine the search and locate the studies. Inclusion criteria of quantitative, qualitative and mixed studies will be applied, in titles, abstract and full text; Two reviewers will independently extract the information using a screening tool; finally, the results will be presented narratively and tables or figures will be used. Ethics and disclosure: This scoping review will involve a secondary analysis of the data already collected and therefore does not require ethical approval. The results of this research will be submitted to peer-reviewed journals for publication and will also be disseminated at conferences and seminars.
\end{abstract}

Keywords: Parenting; Feeding behavior; Caregivers; Child; Qualitative research.

\section{Resumo}

Introdução: A responsabilidade parental dos pais em relação à alimentação dos filhos implica ter um trabalho responsável na seleção, preparação e oferta de alimentos aos filhos; além disso, qualidades de comportamento exemplar, como comportamento alimentar saudável que influencia os hábitos que seus filhos adquirem; no entanto, existem vários fatores determinantes que o afetam negativamente. Objetivo: Identificar os fatores determinantes na responsabilidade parental dos cuidadores em promover o comportamento alimentar de crianças de 6 meses a 12 anos. Métodos: A revisão será realizada de acordo com a metodologia proposta por Hilary Arksey e Lisa O`Malley atualizada pelo The Joanna Briggs Institute (JBI) para análise de escopo. Uma estratégia de busca abrangente será aplicada com o apoio do bibliotecário pesquisador para refinar a busca e localizar os estudos. Serão aplicados critérios de inclusão de estudos quantitativos, qualitativos e mistos, em títulos, resumo e texto completo; dois revisores extrairão independentemente as informações usando uma ferramenta de triagem; finalmente os resultados serão apresentados de forma narrativa e serão utilizadas tabelas ou figuras. Ética e divulgação: Esta revisão de escopo envolverá uma análise secundária dos dados já coletados e, portanto, não requer aprovação ética. Os resultados desta 
pesquisa serão submetidos a periódicos revisados por pares para publicação e também serão divulgados em conferências e seminários.

Palavras-chave: Poder familiar; Comportamento alimentar; Cuidadores; Criança; Pesquisa qualitativa.

\section{Resumen}

Introducción: La responsabilidad parental de los padres respecto a la alimentación de los niños, implica tener un trabajo responsable al seleccionar, preparar y ofrecer alimentos a sus hijos; además, cualidades de comportamiento ejemplar como una conducta alimentaria saludable que influya en los hábitos que sus hijos adquieran; sin embargo, existen múltiples factores determinantes que afectan negativamente. Objetivo: Identificar los factores determinantes en la responsabilidad parental de cuidadores para promover una conducta alimentaria en niños de 6 meses a 12 años. Métodos: La revisión se realizará de acuerdo a la metodología propuesta por Hilary Arksey y Lisa O`Malley actualizada por The Joanna Briggs Institute (JBI) para scoping review. Se aplicará una estrategia de búsqueda integral con el apoyo de bibliotecario de investigación para refinar la búsqueda y localizar los estudios. Se aplicarán criterios de inclusión de estudios cuantitativos, cualitativos y mixtos, en títulos, resúmen y texto completo; dos revisores extraeran la información de manera independiente mediante herramienta de detección; finalmente los resultados se presentarán de forma narrativa y se utilizarán tablas o figuras. Ética y divulgación: Esta scoping review implicará un análisis secundario de los datos ya recopilados y por lo tanto, no requiere aprobación ética. Los resultados de esta investigación se enviarán a revistas revisadas por pares para su publicación y también se difundiran en conferencias y seminarios.

Palabras clave: Responsabilidad parental; Conducta alimentaria; Cuidadores; Niño; Investigación cualitativa.

\section{Introduction}

Alimentación es el conjunto de procesos biológicos, psicológicos y sociológicos relacionados con el consumo de alimentos, es una actividad que las personas realizan varias veces al día y mediante estas acciones proveen al cuerpo humano energía y nutrimentos; asimismo, se satisfacen necesidades intelectuales, emocionales, estéticas y socioculturales. En este sentido, la conducta alimentaria es un conjunto de conductas que una persona adquiere y están determinadas por la repetición de actos relacionados con la selección, preparación y consumo de alimentos; estas respuestas asociadas al acto de alimentarse, modos de alimentarse, padrón rítmico como horarios y duración de la alimentación estan influenciados por las condiciones sociales, económicas y culturales de la familia en un contexto o región determinada Descriptores en Ciencias de la Salud (DeSC (2019a), NOM-043-SSA2 (2012a).

La alimentación se considera correcta ${ }^{1}$ cuando la dieta cumple con las necesidades específicas de las diferentes etapas de la vida, promueve un crecimiento y desarrollo adecuados, permite conservar o alcanzar el peso esperado para la talla y previene el desarrollo de enfermedades. La dieta es un conjunto de alimentos que se consumen cada día y constituye la unidad de la alimentación; estos alimentos pueden ser sólidos y líquidos; debería ser completa y contener todos los nutrimentos e incluir alimentos de los tres grupos, verduras y frutas, cereales, leguminosas y alimentos de origen animal; equilibrada que los nutrimentos guarden las proporciones apropiadas entre sí; inocua exenta de microorganismos patógenos, toxinas, contaminantes y se consuma con moderación; suficiente que cubra las necesidades de todos los nutrimentos para que un adulto tenga una buena nutrición y peso saludable, los niños que crezcan y se desarrollen de manera correcta; variada al incluir diferentes alimentos de cada grupo en las comidas, adecuada acorde con los gustos y la cultura de quien la consume y ajustada a sus recursos económicos DeSC (2019b), NOM-043-SSA2 (2012b).

En México prevalecen enfermedades derivadas de malnutrición, entre estas la desnutrición crónica por deficiencia de micronutrientes, o consumo excesivo de macronutrientes provocando sobrepeso y obesidad en niñas y niños pequeños dejándoles secuelas irreversibles aún después de tratamiento; estas condiciones aumentan el riesgo de diabetes, hipertensión arterial, padecimientos ortopédicos y otras que se asocian a mayor riesgo de infecciones, mayores complicaciones, hospitalizaciones y muerte por COVID-19, (Organización Mundial de la Salud [OMS], 2018a); Seidu, Gillies, Zaccardi,

\footnotetext{
${ }^{1}$ Alimentación correcta es el termino utilizado en Normas Oficiales de México como sinónimo de alimentación saludable.
} 
Kunutsor, Hartmann-Boyce, Yates, Singh, Davies, \& Khunti. (2020); Petrova, Salamanca, Rodríguez, Navarro, Jiménez, \& Sánchez. (2020); (Instituto Nacional de Salud Pública [INSP], 2020a). Además, se prevé que la crisis económica aumentará la inseguridad alimentaria en poblaciones vulnerables, lo que favorecerá el consumo de alimentos ricos en energía, azúcar, sal y grasa y de baja calidad nutricional; también, los altos precios de alimentos provocarán que algunas familias disminuyan la cantidad de alimentos frescos como verduras y frutas. La malnutrición es un problema grave que suele iniciarse desde las primeras horas de vida con la lactancia materna no exclusiva y a partir de los 6 meses se continúa la alimentación complementaria inadecuada y así, toda nuestra vida de los menores puede estar marcada por una alimentación saludable o no saludable; por lo cual es necesario enfatizar que una dieta saludable previene la malnutrición, enfermedades como la obesidad, diabetes, entre otras (OMS, 2018b).

La nutrición es fundamental en todos los seres humanos, favorece un crecimiento sano, mejora la calidad de vida, el desarrollo cognitivo, la autoestima, reduce el riesgo de fracturas y efectos psicológicos por discriminación o bullying; además, la ingesta de vitaminas y minerales benefician el sistema inmunológico lo que contribuye a prevenir enfermedades y proporcionar beneficios a largo plazo al reducir el riesgo de enfermedades en la edad adulta. (8-11). Ribeiro, Garcia, Dametto, Assunção, \& Maciel. (2020); Pakpour, Chen, Lin, Strong, Tsai, \& Lin. (2019); (OMS, 2020a). Sin embargo, en México para 2018, había un 35.6\% de niños entre 5 y 11 años con sobrepeso y obesidad, de ellos un $85.7 \%$ consume bebidas endulzadas no lácteas, un $64.6 \%$ botanas, dulces y postres, el $52.9 \%$ cereales dulces; caso contrario con el consumo de verduras sólo el 22\% las consume y sólo el $43.5 \%$ come frutas (Encuesta Nacional de Salud y Nutrición [ENSANUT], 2018a).

Es trascendental que todas las personas lleven una alimentación saludable para prevenir enfermedades derivadas de la malnutrición por deficiencia de vitaminas y minerales o consumo excesivo de hidratos de carbono, proteinas, grasas, bebidas endulzadas y alimentos con exceso de sodio OMS (2018c), Seidu et al., (2020); Petrova et al., (2020); INSP (2020b). Estas enfermedades tendrán serias repercusiones en el sistema de salud y en la economía mundial, causarán millones de muertes, reducción de la fuerza laboral, las personas enfermas serán poco productivas y tendrán menos probabilidades de estar empleadas, lo que supondrá cerca del $8.9 \%$ del gasto en salud por año y por ende le restará al PIB mexicano cerca de 5.3 puntos porcentuales (Organisation for Economic Cooperation and Developmen [OECD], 2019).

Uno de los objetivos de desarrollo sostenible establece "Hambre cero" que tiene como propósito que todas las personas y en especial los niños, tengan una alimentación suficiente y nutritiva, en general toda persona tiene derecho a la salud y a la alimentación (Programa de las Naciones Unidas para el Desarrollo [PNUD], 2019); (Naciones Unidas [NU], 2021). En México todo niño tiene derecho a una alimentación nutritiva, suficiente y de calidad, el Estado lo garantizará (Ley General de Salud [LGS], 2020), Constitución Política de los Estados Unidos Mexicanos (2020), los niños deberían disfrutar de un alto nivel de salud mediante una alimentación equilibrada, Ley General de los Derechos de las Niñas, Niños y Adolescentes em México (2019). En este sentido, los padres o representantes legales tienen una labor de suma importancia, la responsabilidad parental debería ser un trabajo responsable al seleccionar, preparar y ofrecer alimentos saludables a sus hijos; además, cualidades de comportamiento ejemplar, la conducta alimentaria de los padres, modos de alimentarse y patrón rítmico de alimentación son prácticas que influyen en los hábitos que sus hijos adquieran NOM-043-SSA2 (2012c).

Los padres son personajes de principal influencia para moldear los hábitos y preferencias alimentarias e influirán en las preferencias hacia ciertos hábitos en sus hijos, Sánchez, Reyes, \& González (2014); Martínez, Petermann, Villagrán, Ulloa, Nazar, Troncoso, Garrido, Mardones, Lanuza, Leiva, Lasserre, Martorell, \& Celis (2020), particularmente materno filial por su implicación en los hábitos y cuidados en alimentación a través de su rol esencial en la crianza de sus hijos; igualmente, la influencia familiar está determinada por el conocimiento en alimentación, Martos, Martínez, González, \& Argente (2019); Encina, Gallegos, Espinoza, Arredondo \& Palacios (2019); Silventoinen \& Konttinen (2020). Se recomienda que los padres 
tengan un estilo de vida saludable y lo fomenten, los niños aprenderán un modelo de dieta y conducta alimentaria de los padres, lo que influirá en el estado nutricional; además, genéticamente los padres heredan un IMC elevado, OMS (2020b); Moreno \& Galindo (2015); Silventoinen\& Konttinen (2020); Blanco, Veiga, Sepúlveda, Izquierdo, Román, López \& Rojo (2020). La obesidad materna es un predictor de obesidad en hijos, existe correlación entre factores genéticos de obesidad particularmente materno filial y aumenta el riesgo de enfermedades en la edad adulta, Martínez et al., (2020); Martos., et al (2019); Blanco et al., (2020); Arrocha, Rodríguez \& Pedroso (2020). En este sentido, el desempeño de los padres al ofrecer y otorgar alimentos a los hijos es responsabilidad de los padres, representantes legales que ejercen la patria potestad hasta que cumplan la mayoría de edad. El objetivo de esta scoping review será identificar los factores determinantes en la responsabilidad parental de cuidadores para promover una conducta alimentaria en niños de 6 meses a 12 años.

Se realizó una búsqueda preliminar en COCHRANE, PROSPERO, BMC, en BJI de revisiones sistemáticas y scoping review del tema responsabilidad parental de cuidadores y conducta alimentaria de niños. Se han realizado revisiones que abordan trastornos de alimentación e intervenciones de prevención en niños y adolescentes, intervenciones conductuales de dieta y actividad física, Martínez, Rubio, Reche, Leyva, Hernández, Hernández, Martínez (2020); Chua, Tam, \& Shorey (2020); Qian, Hu, Wan, Li, Wu, Ren, \& Yu (2013); Al, Loveman, Colquitt, Mead, Johnson, Fraser, Olajide, Murphy, Velho, O'Malley, Azevedo, Ells, Metzendorf, \& Rees (2017); Mead, Brown, Rees, Azevedo, Whittaker, Jones, Olajide, Mainardi, Corpeleijn, O'Malley, Beardsmore, Al-Khudairy, Baur, Metzendorf, Demaio, \& Ells (2017); Pratt, \& Woolfenden (2002); Perkins, Murphy, Schmidt, \& Williams (2006). Además, experiencias de los cuidadores al alimentar a niños, comportamientos de pica, acceso a los alimentos, efectividad de intervenciones basadas en la familia, intervenciones dietéticas maternas para mejorar la salud materno infantil, sobrepeso y obesidad en niños, imagen corporal, actitudes, comportamientos y conocimientos alimentarios de los padres Taylor, Zhang, Foster, Novak, \& Badawi (2018); Shi, Lukomskyj, \& Allman (2020); Clark, \& Williams (2020); Chai, Collins, May, Brain, Wong See, \& Burrows (2019); Ng, Kaur, Koo, \& Mukhtar (2020); Watterson, MacDonald Collins, Hutchesson, Shrewsbury, Vincze, Heslehurst, \& Follong (2018). Ho, Jensen, Burrows, Neve, Garnett, Baur, Stewart, Collins (2013); Collins, Neve, Morgan, Fletcher, Williams, Young, Callister (2013); Damiano, Hart, Paxton, Cornell, \& Sutherland (2014). No se han realizado revisiones que vinculen la responsabilidad parental de cuidadores y la conducta alimentaria de cuidadores de niños.

\section{Methodology}

La scoping review se realizará de acuerdo a la metodología propuesta por Hilary Arksey y Lisa O`Malley actualizada por The Joanna Briggs Institute (JBI) se efectuará de noviembre 2021 a febrero 2022, la estrategia de búsqueda en tres pasos descritos por Peters, Godfrey, McInerney, Munn, Tricco, Khalil (2020), primero se diseñará la búsqueda con apoyo de bibliotecario de investigación o científico de la información para garantizar un enfoque riguroso con el objetivo de realizar una busqueda exhaustiva de estudios y minimizar el sesgo de selección. Los resultados de la búsqueda se informarán a través de la metolodogía PRISMA-ScR Tricco, Lillie, Zarin, O'Brien, Colquhoun, Levac, et al., (2018).

\section{Etapa 1: Definición de la Pregunta/pregunta de investigación}

A través de la discusión entre los investigadores y los interesados, la pregunta de investigación se definió como ¿Cuáles son los factores determinantes en la responsabilidad parental de cuidadores para promover una conducta alimentaria saudable en niños de 6 meses a 12 años? A partir de la pregunta general, se definieron las siguientes preguntas específicas de investigación:

1. ¿Cómo la responsabilidad parental de cuidadores ha influido en la conducta alimentaria de niños de 6 meses a 12 años? 
2. ¿Cómo la conducta alimentaria de cuidadores ha influido en la conducta alimentaria de sus hijos de 6 meses a 12 años?

3. ¿Cómo diversos autores han analizado la responsabilidad parental de cuidadores sobre la conducta de alimentación de sus hijos de 6 meses a 12 años?

\section{Etapa 2: desarrollar los criterios de inclusión y exclusión}

\section{Criterios de inclusión}

Estos se definirán en función de la estructura, el concepto y los contextos de la población (PCC):

Population P: Cuidadores familiares como madre, padre de niños de 6 meses a 12 años.

Concept C: Factores determinantes, responsabilidad parental, conducta alimentaria.

Context C: Todos los contextos geográficos.

\section{Participantes}

- Esta revisión incluirá estudios con enfoque cuantitativo como ensayos controlados aleatorios (ECA) cuasiexperimentales con y sin grupo control, ensayo clínico que presenten resultados aplicados a cuidadores padre, madre e hijos.

- Estudios con enfoque cualitativos como etnografía, fenomenología, teoría fundamentada, investigación acción y otros aplicados a cuidadores familiares padre, madre e hijos.

- Estudios mixtos aplicados a cuidadores familiares padre, madre e hijos.

\section{Concepto}

- Estudios primarios que midieron las variables responsabilidad parental y conducta alimentaria desde enfoque cuantitativo, cualitativo o mixto.

- Estudios que analizaron los conceptos responsabilidad parental y conducta alimentaria.

\section{Contexto}

- Estudios realizados en todos los contextos geográficos dentro y fuera de la vivienda.

- Todos los idiomas, sin límite en tiempo.

\section{Tipos de fuentes de evidencia}

- Ensayos controlados aleatorios ECA, cuasiexperimentales con y sin grupo control, ensayo clínico, estudios cualitativos como etnografía, fenomenología, teoría fundamentada, investigación acción.

\section{Criterios de exclusión}

Books and documents (libros y documentos), meta-analysis (metaanálisis), review (artículos de revisión), syntematic review (revisiones sistemáticas), artículos de opinión, disertaciones, análisis de concepto.

\section{Etapa 3: Estrategia de investigación/búsqueda}

Se definieron los terminos MeSH: responsabilidad parental (parenting) (poder familiar); conducta alimentaria (feeding behavior) (comportamento climentar); cuidadores (caregivers) (cuidadores); niño (child) (criança). 
Inclusión: todos los idiomas, sin límite en tiempo, ensayos controlados aleatorios (ECA), cuasiexperimentales con y sin grupo control, ensayo clínico, estudios cualitativos como etnografía, fenomenología, teoría fundamentada, investigación acción.

Filtros, criterios de eliminación: books and documents (libros y documentos), meta-analysis (metaanálisis), review (artículos de revisión), syntematic review (revisiones sistemáticas), artículos de opinión, disertaciones, análisis de concepto.

La búsqueda inicial será en PubMed.gov y CINAHL (EBSCO). Análisis de palabras en el título y resumen de artículos recuperados. Segunda búsqueda utilizando todas las palabras clave identificadas y los términos del índice en todas las bases de datos incluidas, lectura de texto completo de artículos que cumplieron los criterios de elegibilidad. Se buscarán fuentes adicionales en la lista de referencias de artículos y documentos incluidos en la revisión; además, búsqueda en literatura gris.

La búsqueda completa se realizará en las bases de datos electrónicas: MEDLINE (Ovid), PubMed.gov, CINAHL(EBSCO), EMBASE (Elsevier), SCOPUS, LILACS, WEB OF SINCE, Portal Regional de la (BVS), Wiley Online Library (The Journal of Advanced Nursing (JAN)), literatura gris como tesis que aborden la responsabilidad parental, conducta alimentaria de cuidadores familiares como madre, padre de niños de 6 meses a 12 años; en todos los contextos geográficos, se aplicará la estrategia de búsqueda diseñada con apoyo de bibliotecario de investigación.

\section{Fuente de análisis y selección de pruebas}

Posterior a la búsqueda, los artículos identificados se recopilarán y se eliminarán los duplicados. Los procedimientos serán realizados por dos investigadores independientes que se reunirán para llegar a un consenso de integración de artículos y en los casos de desacuerdo sobre la inclusión o exclusión en la revisión habrá un tercer revisor que conciliará y tomará la decisión final. Los dos revisores de manera independiente identificarán los estudios que cumplan con los criterios de inclusión, revisarán los títulos y resúmenes de acuerdo a los criterios; los artículos destacados se recuperarán en texto completo y se importarán al sistema JBI para la gestión unificada, evaluación y revisión. Se informarán las razones de exclusión de artículos de texto completo. Los resultados de la búsqueda se informarán a través de la metodología PRISMA-ScR, para los estudios cualitativos se aplicará la lista de verificación de evaluación crítica de JBI para investigación cualitativa Tricco et al., (2018).

\section{Extracción de datos}

Los datos se extraerán de los artículos por dos revisores de manera independiente y se utilizará herramientas como tablas y gráficos para la extracción de datos que será elaborada previamente por los revisores tomando en cuenta los criterios de inclusión, serán extraidos datos sobre título del artículo, autor/año, país, título, objetivo, tipo de estudio, participantes (características/ número total) criterios de inclusión, concepto datos de las fuentes de evidencia incluidas en relación con el concepto, contexto describiendo todos los contextos geográficos para identificar factores culturales, sociales, económicos, étnico, hallazgos relevantes relacionados. Los datos para esta revisión se obtendrán de literatura pública de acceso abierto y los resultados se difundirán a través de un acceso abierto y se respetarán los derechos de autor y terminos de licencia de las bases de datos.

\section{Análisis y presentación de resultados}

Los resultados se presentarán en síntesis narrativa o descriptiva utilizando tablas o un mapa de los datos extraídos y que respondan la pregunta de investigación describiendo lógicamente los objetivos, características de los participantes, conceptos y contextos que cumplan con el objetivo de revisión. Los resultados de la búsqueda se informarán y se presentarán en un diagrama de flujo de elementos de informe a través de la metodología PRISMA-ScR Tricco et al., (2018). Se incluirán 
las fuentes de evidencia que se seleccionaron para su inclusión al análisis describiendo los resultados de la búsqueda, eliminación de duplicados, recuperación completa y presentación final; se incluirá una tabla general con las fuentes de evidencia incluidas. En los resultados se discutirán con necesidades de investigación identificadas en el análisis.

\section{Results and Discussion}

Se discutirán los resultados de la revisión en el contexto de la literatura, la práctica de enfermería y las políticas actuales para comprender los determinantes sociales que influyen en un problema de salud multifactorial; además, se mencionarán las limitaciones encontradas.

\section{References}

Arrocha, M. F., Rodríguez, C. F., Pedroso, M. T. G. (2020). Risk factors associated with overweight and obesity in school-age children. Revista Cubana de Pediatria; 92(2):1-11. http://scielo.sld.cu/scielo.php?script=sci_arttext\&pid=S0034-75312020000200004\&lng=es\&tlng=es.

Al-Khudairy, L., Loveman, E., Colquitt, J. L., Mead, E., Johnson, R. E., Fraser, H., Olajide, J., Murphy, M., Velho, R. M., O'Malley, C., Azevedo, L. B., Ells, L. J., Metzendorf, M. I., \& Rees, K. (2017). Diet, physical activity and behavioural interventions for the treatment of overweight or obese adolescents aged 12 to 17 years. The Cochrane database of systematic reviews, 6(6), CD012691. https://doi.org/10.1002/14651858.CD012691.

Blanco, M., Veiga, O. L., Sepúlveda, A. R., Izquierdo-Gomez, R., Román, F. J., López, S., \& Rojo, M. (2020). Ambiente familiar, actividad física y sedentarismo en preadolescentes con obesidad infantil: estudio ANOBAS de casos-controles. Atención primaria.52(4): 250-257. https://doi.org/10.1016/j.aprim.2018.05.013.

Clark, O. M., \& Williams, R. (2020). Pica behaviors in pediatric patients with sickle cell disease: a scoping review protocol. JBI evidence synthesis. 18(9): 2018-2024. https://doi.org/10.11124/JBISRIR-D-19-00241.

Collins, C., Neve, M., Morgan, P., Fletcher, K., Williams, R., Young, M., Callister, R. (2013). Effectiveness of interventions with a dietary component on weight loss maintenance: a systematic review. JBI Database. 11(8):317-414. doi: 10.11124/jbisrir-2013-708.

Constitución Política de los Estados Unidos Mexicanos. Cámara de Diputados del H. Congreso de la Unión. Diario Oficial de la Federación: https://bit.ly/2SW7OEZ.

Chai, L. K., Collins, C., May, C., Brain, K., Wong See, D., \& Burrows, T. (2019). Effectiveness of family-based weight management interventions for children with overweight and obesity: an umbrella review. JBI database of systematic reviews and implementation reports. 17(7): 1341-1427. https://doi.org/10.11124/JBISRIR-2017-003695

Chua, J., Tam, W., \& Shorey, S. (2020). Research Review: Effectiveness of universal eating disorder prevention interventions in improving body image among children: a systematic review and meta-analysis. Journal of child psychology and psychiatry, and allied disciplines. 61(5): 522535. https://doi.org/10.1111/jcpp.13164.

Damiano, S. R., Hart, L., Paxton, S. J., Cornell, C., \& Sutherland, F. (2014). Are body image and eating attitudes, behaviours, and knowledge of parents of pre-schoolers associated with parent feeding practices? Journal of Eating Disorders. 2(Suppl 1): O34. https://doi.org/10.1186/2050-2974-2-S1-O34.

DeSC. (2019). Conducta alimentaria. Descriptores en Ciencias de la Salud: http://decs.bvsalud.org/E/homepagee.htm.

Encina, V. C., Gallegos, L. D., Espinoza, M. P., Arredondo G. D \& Palacios C. K. (2019). Comparación de la conducta alimentaria en niños de diferentes establecimientos educacionales y estado nutricional. Revista chilena de nutrición. 46(3): 254-263. https://dx.doi.org/10.4067/S0717-75182019000300254.

ENSANUT. (2018a). Informe de Resultados. Encuesta Nacional de Salud y Nutrición: https://ensanut.insp.mx/encuestas/ensanut2018/informes.php.

Ho, M., Jensen, M.E., Burrows, T., Neve, M., Garnett, S.P., Baur, L., Stewart, L., Collins, C. (2013). Best practice dietetic management of overweight and obese children and adolescents: a 2010 update of a systematic review. JBI Database. 11(10): 190-293. DOI: 10.11124/jbisrir-2013-890.

INSP. (2021b). Prevención de mala nutrición en niñas y niños en México ante la pandemia de COVID-19: Recomendaciones dirigidas a tomadores de decisiones. Instituto Nacional de Salud Pública: https://www.insp.mx/informacion-relevante/impacto-del-covid-19-en-la-mala-nutricion-infantil.

INSP. (2020a). Recomendaciones de política pública nacional, estatal y local para la prevención, control y reducción de la mala nutrición en niñas, niños y adolescentes en México. Instituto Pacional de https://www.insp.mx/assets/pdfjs/web/viewer.html?file=https://www.insp.mx/resources/images/stories/2021/docs/210129_recomendaciones_malanutricionwe bok.pdf.

Ley General de Salud México. (2020). Cámara de Diputados del Honorable Congreso de la Unión. Diario Oficial de la Federación: http://www.diputados.gob.mx/LeyesBiblio/pdf/142_240120.pdf.

Ley General de los Derechos de Niñas, Niños y Adolescentes. (2019). Cámara de Diputados Honorable Congreso de la Unión México: https://bit.ly/3iQu65y.

Martínez-Olcina, M., Rubio-Arias, J.A., Reche-García, C., Leyva-Vela, B., Hernández-García, M., Hernández-Morante, J.J., Martínez-Rodríguez, A. (2020). Eating Disorders in Pregnant and Breastfeeding Women: A Systematic Review. Medicina. 56(7):352. doi:10.3390/medicina56070352. 
Martínez-Sanguinetti, M. A., Petermann-Rocha, F., Villagrán, M., Ulloa, N., Nazar, G., Troncoso-Pantoja, C., Garrido-Méndez, A., Mardones, L., Lanuza, F., Leiva, A. M., Lasserre-Laso, N., Martorell, M \& Celis-Morales, C. (2020). Desde una mirada global al contexto chileno: ¿Qué factores han repercutido en el desarrollo de obesidad en Chile? (Parte 2). Revista chilena de nutrición. 47(2): 307-316. https://dx.doi.org/10.4067/S0717-75182020000200307.

Martos-Moreno, G. Á., Martínez-Villanueva, J., González-Leal, R., \& Argente, J. (2019). Importancia de los factores socioeconómicos en estudios de obesidad. Repuesta de los autores [The importance of socioeconomic factors in obesity studies. Authors reply]. Anales de pediatria.91(6): 424. https://doi.org/10.1016/j.anpedi.2019.06.007.

Moreno, J.M., Galindo, M.J. (2015). Alimentación del niño preescolar, escolar y del adolescente. Pediatría Integral. XIX (4): 268-276. Available from: https://bit.ly/3nWd4WB.

Mead, E., Brown, T., Rees, K., Azevedo, L. B., Whittaker, V., Jones, D., Olajide, J., Mainardi, G. M., Corpeleijn, E., O'Malley, C., Beardsmore, E., AlKhudairy, L., Baur, L., Metzendorf, M. I., Demaio, A., \& Ells, L. J. (2017). Diet, physical activity and behavioural interventions for the treatment of overweight or obese children from the age of 6 to 11 years. The Cochrane database of systematic reviews. 6(6): CD012651. https://doi.org/10.1002/14651858.CD012651.

NOM-043-SSA2. (2012). Servicios básicos de salud. Promoción y educación para la salud en materia alimentaria. Norma Oficial Mexicana: https://bit.ly/3dgWVHg.

NU. (2021). La Declaración Universal de Derechos de Humanos. Naciones Unidas: https://www.un.org/es/about-us/universal-declaration-of-human-rights.

Ng, C. M., Kaur, S., Koo, H. C., \& Mukhtar, F. (2020). Nutrition-related outcomes of children's involvement in healthy meal preparation: a scoping review protocol. JBI evidence synthesis. 18(3): 534-542. https://doi.org/10.11124/JBISRIR-D-19-00209.

OMS. (2018 a,b,c). Alimentación Sana. Datos y cifras. Organización Mundial de la Salud: https://www.who.int/es/news-room/fact-sheets/detail/healthy-diet. OMS. (2020a). Obesidad y sobrepeso. Organización Mundial de la Salud: https://bit.ly/36PQQ3k.

OMS. (2020b). El papel de los padres. Organización Mundial de la Salud: https://www.who.int/dietphysicalactivity/childhood_parents/es/.

OECD. (2019). La Pesada Carga de la Obesidad: La Economía de la Prevención. Organisation for Economic Cooperation and Developmen: https://www.oecd.org/about/secretary-general/heavy-burden-of-obesity-mexico-january-2020-es.htm.

Pratt, B. M., \& Woolfenden, S. R. (2002). Interventions for preventing eating disorders in children and adolescents. The Cochrane database of systematic reviews. 2002(2): CD002891. https://doi.org/10.1002/14651858.CD002891.

Perkins, S. J., Murphy, R., Schmidt, U., \& Williams, C. (2006). Self-help and guided self-help for eating disorders. The Cochrane database of systematic reviews. (3): CD004191. https://doi.org/10.1002/14651858.CD004191.pub2.

Pakpour, A. H., Chen, C. Y., Lin, C. Y., Strong, C., Tsai, M. C., \& Lin, Y. C. (2019). Relación entre el sobrepeso en niños y calidad de vida: comparación entre Sizing Me Up, PedsQL y Kid-KINDL. International Journal of Clinical and Health Psychology. 19(1): 49-56. https://doi.org/10.1016/j.ijchp.2018.06.002

PNUD. (2019). Objetivos de Desarrollo Sostenible. Programa de las Naciones Unidas para el Desarrollo: https://www.undp.org/content/undp/es/home/sustainable-development-goals/goal-2-zero-hunger.html.

Petrova, D., Salamanca-Fernández, E., Rodríguez Barranco, M., Navarro Pérez, P., Jiménez Moleón, J. J., \& Sánchez, M. J. (2020). La obesidad como factor de riesgo en personas con COVID-19: posibles mecanismos e implicaciones. Atencion primaria. 52(7): 496-500. https://doi.org/10.1016/j.aprim.2020.05.003.

Peters, M.D.J., Godfrey, C., McInerney, P., Munn, Z., Tricco, A.C., Khalil, H. (2020). Chapter 11: Scoping Reviews. In: Aromataris E, Munn Z (Editors). JBI Manual for Evidence Synthesis, JBI. Available from: https://synthesismanual.jbi.global.

Qian, J., Hu, Q., Wan, Y., Li, T., Wu, M., Ren, Z., \& Yu, D. (2013). Prevalence of eating disorders in the general population: a systematic review. Shanghai archives of psychiatry. 25(4): 212-223. https://doi.org/10.3969/j.issn.1002-0829.2013.04.003.

Ribeiro, K., Garcia, L., Dametto, J., Assunção, D., \& Maciel, B. (2020). COVID-19 and Nutrition: The Need for Initiatives to Promote Healthy Eating and Prevent Obesity in Childhood. Childhood obesity (Print). 16(4): 235-237. https://doi.org/10.1089/chi.2020.0121.

Sánchez-García, R., Reyes-Morales, H, \& González-Unzaga, M. A. (2014). Preferencias alimentarias y estado de nutrición en niños escolares de la Ciudad de México. Boletín médico del Hospital Infantil de México. 71(6): 358-366. https://doi.org/10.1016/j.bmhimx.2014.12.002.

Silventoinen, K., \& Konttinen, H. (2020). Obesity and eating behavior from the perspective of twin and genetic research. Neuroscience and biobehavioral reviews. 109:150-165. https://doi.org/10.1016/j.neubiorev.2019.12.012.

Seidu, S., Gillies, C., Zaccardi, F., Kunutsor, S. K., Hartmann-Boyce, J., Yates, T., Singh, A. K., Davies, M. J., \& Khunti, K. (2020). The impact of obesity on severe disease and mortality in people with SARS-CoV-2: A systematic review and meta-analysis. Endocrinology, diabetes \& metabolism. 4(1): e00176. https://doi.org/10.1002/edm2.176.

Shi, Y., Lukomskyj, N., \& Allman-Farinelli, M. (2020). Food access, dietary acculturation, and food insecurity among international tertiary education students: a scoping review protocol. JBI evidence synthesis. 18(9): 2090-2097. https://doi.org/10.11124/JBISRIR-D-19-00328.

Taylor, C., Zhang, M., Foster, J., Novak, I., \& Badawi, N. (2018). Caregivers' experiences of feeding children with cerebral palsy: a systematic review protocol of qualitative evidence. JBI database of systematic reviews and implementation reports. 16(3): 589-593. https://doi.org/10.11124/JBISRIR-2017003521 .

Tricco, AC., Lillie, E., Zarin, W., O'Brien, K.K., Colquhoun, H., Levac, D., et al. (2018). PRISMA Extension for Scoping Reviews (PRISMAScR): Checklist and Explanation. Ann Intern Med.169: 467-473 doi: 10.7326/M18-0850.

Watterson, C., MacDonald-Wicks, L., Collins, C., Hutchesson, M., Shrewsbury, V., Vincze, L., Heslehurst, N., \& Follong, B. (2018). Effectiveness of maternal dietary interventions for improving mother and infant health outcomes: an umbrella review protocol. JBI database of systematic reviews and implementation reports. 16(10): 1929-1938. https://doi.org/10.11124/JBISRIR-2017-003653. 